\title{
Actitud ante la inclusión de niños y niñas con necesidades educativas especiales Consideraciones de padres, madres y profesionales de la educación
}

Anna-Jordina Llorens Ferrer al198570@uji.es 
I. Resumen

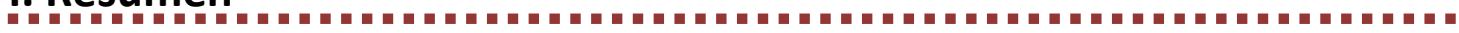

En el presente trabajo realizamos una exploración sobre las actitudes de padres, madres y profesionales de la educación hacia la inclusión de alumnos y alumnas con Necesidades Educativas Especiales (NEE) en aulas ordinarias. El objetivo es analizar si hay diferencias en sus actitudes y si éstas están influidas por variables como estudios realizados, género, profesión, conocimiento de las dificultades, etc. Para ello, hemos pasado varios cuestionarios sobre actitudes a una muestra compuesta por 33 maestros de educación primaria y secundaria, 22 padres y 26 madres de alumnos y alumnas.

De entre los resultados encontrados, destacamos que existe una actitud positiva general hacia la inclusión de este colectivo de alumnos, aunque ésta no funciona de forma óptima debido, fundamentalmente, a la falta de recursos y estrategias adecuadas.

Palabras clave: Inclusión, Necesidades Educativas Especiales, actitudes, padres y madres, profesores.

\section{Introducción}

La educación inclusiva e integradora es un movimiento de ámbito mundial que ha ido cobrando importancia en los últimos años, especialmente desde que en 1994 la Declaración de Salamanca proclamara la inclusión como medio más eficaz para educar a todos los niños/as y jóvenes en el sistema educativo ordinario, con independencia de sus diferencias o dificultades individuales y sociales (UNESCO, 1994).

En esta «Conferencia Mundial de Salamanca sobre Necesidades Educativas Especiales. Acceso y Calidad" (más conocida como Declaración de Salamanca) el principio fundamental es el de «que todos los alumnos/as aprendan juntos, siempre que sea posible, independientemente de las dificultades y de las diferencias que presenten", proclamando igualmente que "las escuelas regulares siguiendo una orientación inclusiva constituyen los medios más capaces para combatir las actitudes discriminatorias fomentando comunidades abiertas y solidarias, construyendo una sociedad inclusiva y procurando una escuela para todos..."

Para autores como Stainback y Stainback (1990) la escuela inclusiva debe servir para ofrecer a cada estudiante la posibilidad de aprender a vivir y trabajar con sus iguales en contextos naturales, de educación integrada y comunidad, para evitar los efectos inherentes a la segregación cuando los estudiantes están en lugares separados, en aulas o centros de educación especial, para hacer lo que es justo y equitativo. 
Es decir, en términos pedagógicos se estima que debe ofrecerse una igualdad de oportunidades cuando se reconoce el derecho a la diferencia y al pluralismo cultural y cuando existen mecanismos de individualización de acuerdo a las necesidades de los alumnos, así como cuando se realiza un aprendizaje diferenciado (Falcao, 1992; Correia, 1997).

De este modo la escuela inclusiva se enmarcaría dentro de un nuevo modelo llamado a ser «un lugar donde los aprendizajes son posibles cualquiera que fuese el tipo de deficiencia; es también un modelo de escuela que se centra en el niño miembro de la comunidad y, protagonista de su proceso de aprendizaje, valorando sus éxitos en lugar de sus fracasos» (Dueñas, 1991: 52).

Desde la Declaración de Salamanca, el objetivo de todos aquellos países que se adhirieron a las declaraciones internacionales, entre ellos España, ha sido desarrollar escuelas que respondan con éxito a las necesidades educativas de todo el alumnado.

Es preciso señalar que el concepto de Necesidades Educativas Especiales (NEE) no sólo involucra a alumnos/as que presenten alguna discapacidad notoria, sino que también incluye a aquellos niños que tengan dificultades de aprendizajes, retrasos madurativos, alteraciones emocionales, problemas conductuales, etc. Además en la actualidad se considera que los niños superdotados también presentan una NEE.

Dado que el reto planteado anteriormente depende en gran medida del profesorado, el análisis de sus percepciones, actitudes y expectativas son críticas para el desarrollo exitoso de esta política educativa.

La introducción en las escuelas de políticas educativas cada vez más inclusivas han traído consigo cambios importantes en los roles y responsabilidades de los profesionales de la educación. Estos cambios generalmente no se han visto acompañados de un análisis previo de su pensamiento (creencias y actitudes) hacia este proceso, cosa que puede estar comprometiendo seriamente el desarrollo de la inclusión. Dado que la investigación sobre el pensamiento del profesor demuestra que las percepciones y actitudes definen los procesos de pensamiento, acción y disposición al cambio del profesorado ante determinados retos (Richardson, 1996), el estudio de sus actitudes se convierte en elemento determinante para conocer el desarrollo y comprender mejor el comportamiento instructivo del profesor/maestro en el aula.

De acuerdo a lo anterior, en el ciclo de debates organizado por la UNICEF, UNESCO \& HINEN (2001), sobre la inclusión de niños con discapacidad en la escuela regular, uno de los obstáculos para llevar a buen término el proceso de integración, consistiría en la dificultad para lograr un cambio en las representaciones y creencias que tienen los agentes educativos con respecto a las posibilidades de aprendizaje de las personas con discapacidad. 
Muchos estudios realizados en torno a las representaciones, creencias y actitudes de los profesores frente a la integración escolar, dan cuenta de que aquello que el profesor piensa sobre integración, se verá reflejado en la puesta en marcha que hace de la misma (Parrilla, 1992). Asumir la diversidad, desde sus diferentes dimensiones, como aquellas de tipo cultural, de capacidades, motivaciones y expectativas, desde los más a menos dotados, como es el caso de los alumnos discapacitados, implica un gran reto, debido al conjunto de creencias, representaciones y estereotipos que maneje la sociedad, de las personas discapacitadas, y, por consiguiente, con las concepciones que se va formando también el profesorado. Las representaciones que tienen los individuos sobre determinado hecho, objeto o persona, en este caso el profesorado que trabaja con niños que tienen discapacidad en el aula común, traspasa la actuación pedagógica, dejando entrever lo que piensan o imaginan sobre estos niños. (Damm, 2009).

Las representaciones y creencias que tenga el maestro en relación a los niños con Necesidades Educativas Especiales sin duda alguna que se verán reflejadas en la actitud que evidencie en el aula. $Y$ es precisamente en relación a este aspecto que se ha puesto de manifiesto como uno de los principales obstáculos para llevar a cabo el proceso de integración (Damm, 2009).

El estudio de las percepciones y actitudes de los maestros/profesores hacia la inclusión del alumnado con NEE se ha realizado de una forma global, sin considerar ni prestar suficiente atención a las condiciones en que ésta se está llevando a cabo en los centros educativos (Chiner, 2011). Por ello, con este trabajo queremos profundizar en el análisis de dichas actitudes atendiendo a aquellos condicionantes o factores que pueden determinar en gran medida su éxito o su fracaso.

Además, hemos incluido el estudio de la opinión y actitudes de los padres y madres al respecto, ya que consideramos que el ambiente familiar, junto con el ambiente escolar, son los referentes más importantes en las primeras etapas educativas.

\section{Objetivos}

En nuestro trabajo tenemos como objetivos:

- Analizar las actitudes de los maestros y padres/madres frente a la inclusión del alumnado con NEE en las aulas ordinarias.

- Analizar si estas actitudes están relacionadas con aspectos como: tipo de estudios cursados, si tienen niños/as o próximas a ellos niños/as, familiares o conocidos con NEE, el lugar de trabajo, el colegio al que llevan sus hijos, la edad y el sexo. 
Asimismo, presentamos nuestras hipótesis de partida:

1. A mayor grado de preparación académica, mayor actitud inclusiva.

2. A menor número de habitantes en una población, mayor actitud de inclusión entre los ciudadanos.

3. Aquellos profesores y padres de alumnos de colegios públicos, presentan una actitud más positiva hacia la inclusión.

4. Las mujeres presentan una actitud más inclusiva.

5. A mayor experiencia por parte del profesorado, mayor actitud inclusiva presenta el docente.

\section{Material y método}

\section{Muestra}

Han formado parte de nuestro estudio un total de 81 sujetos, de los cuales 33 son profesores/as, 26 madres de alumnos/as y 22 padres de alumnos/as.

Todos ellos, son profesores/as o padres/madres de alumnos/as cuyas edades están comprendidas entre los 6 y los 18 años. Este rango de edad comprende los 6-12 de la Educación Secundaria Obligatoria (ESO), más los dos años de margen que el sistema educativo permite alargar la escolarización para poder obtener el título de la ESO.

\section{Instrumento}

A los profesores/as que conforman parte de nuestra muestra se les pasó dos cuestionarios. Por un lado, uno respecto a la opinión sobre la inclusión de niños con Necesidades Educativas Especiales (NEE) adaptado de Larrivée y Cook (1979) por Marina Álvarez, Pilar Castro, María Ángeles Campo-Mon y Eva Álvarez-Martino (2005) en su trabajo Actitudes de los maestros ante las necesidades educativas específicas. Este cuestionario se compone de 30 ítems que recogen afirmaciones a las que se ha de contestar en base a una escala Likert de 1 a 5 según su grado de acuerdo o desacuerdo. Los ítems miden actitudes frente a la inclusión de niños/as con NEE en aulas ordinarias.

El segundo cuestionario que se les pasó a los profesores consta de una matriz, donde se presentan ocho ítems que versan sobre la formación/conocimientos que se tiene sobre distintas NEE y el grado de recursos/apoyos que ellos creen que necesitan los alumnos con alguna necesidad en concreto. Estas ocho cuestiones se realizan para cada una de las siguientes necesidades educativas: Dificultades de Aprendizaje/Lectura, Dificultades de Aprendizaje/Matemáticas, Superdotados, Retraso Mental, 
Dificultad Visual, Dificultad Auditiva, Dificultad Motórica, Dificultad en Lenguaje, Trastorno Espectro Autista, Trastorno Déficit Atencional y Problemas de Conducta.

Respecto a los padres, se les administró el cuestionario acerca de la opinión sobre la inclusión de niños con Necesidades Educativas Especiales (NEE) (adaptado de Larrivée y Cook, 1979) que hemos comentado anteriormente (el mismo que se les pasó a los profesores). En el cuestionario para los padres se modificaron varios términos técnicos para facilitar su comprensión lingüística y que fuera un cuestionario accesible para todos ellos que no se dedican al ámbito de la educación. En todo caso las modificaciones fueron mínimas e insignificativas.

\section{Procedimiento}

Una vez preparados los cuestionarios (con las leves modificaciones en el de los padres/madres), se pasó al reparto de los mismos. Para ello, se llevaron personalmente a distintos centros educativos públicos, concertados y privados de la provincia de Castellón y Valencia, a centros particulares y conocidos. En principio los cuestionarios se entregaron tanto en papel como por vía electrónica, a través de Internet, por medio de la opción que facilita Facebook de Encuesta Fácil. Esta opción no dio los resultados esperados, ya que las encuestas recibidas por este medio llegaban con algún ítem sin cumplimentar. Por ello, no se tuvieron en cuenta los cuestionarios que se habían respondido por esta vía y seleccionamos únicamente aquellos facilitados y recogidos en papel que estaban cumplimentados correctamente.

Los cuestionarios se llevaron tanto a los centros como a los particulares, y se les dio de tiempo una semana para cumplimentarlos. Cumplido el periodo, se pasó personalmente a recogerlos. De esta manera, hemos de remarcar que los cuestionarios los cumplimentaron sin nuestra presencia, favoreciendo así que las respuestas fueran lo más sinceras posible.

\section{Análisis de datos}

Una vez recogidos los cuestionarios, se procedió al vaciado de los datos y a su posterior análisis. Para ello se utilizó el paquete estadístico SPSS versión 19. Los análisis realizados son descriptivos: frecuencia de cada valor, máximos, mínimos, medias, medianas, moda, varianza y desviaciones típicas. Aunque nos centramos básicamente en la media de los resultados obtenidos. 


\section{Resultados}

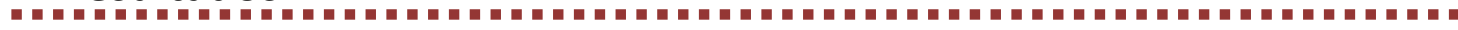

En primer lugar expondremos los resultados de los cuestionarios cumplimentados por padres y madres. Cuando aludimos, únicamente, el término "padres" estamos refiriéndonos a ambos sexos, pero en momentos concretos se hará distinción de los dos sexos (como por ejemplo y más concretamente en el apartado de género) y por ello se especificará la distinción. Los resultados obtenidos son las medias de entre 1 a 5 de puntuación. Las variables son las siguientes:

\section{Estudios realizados}

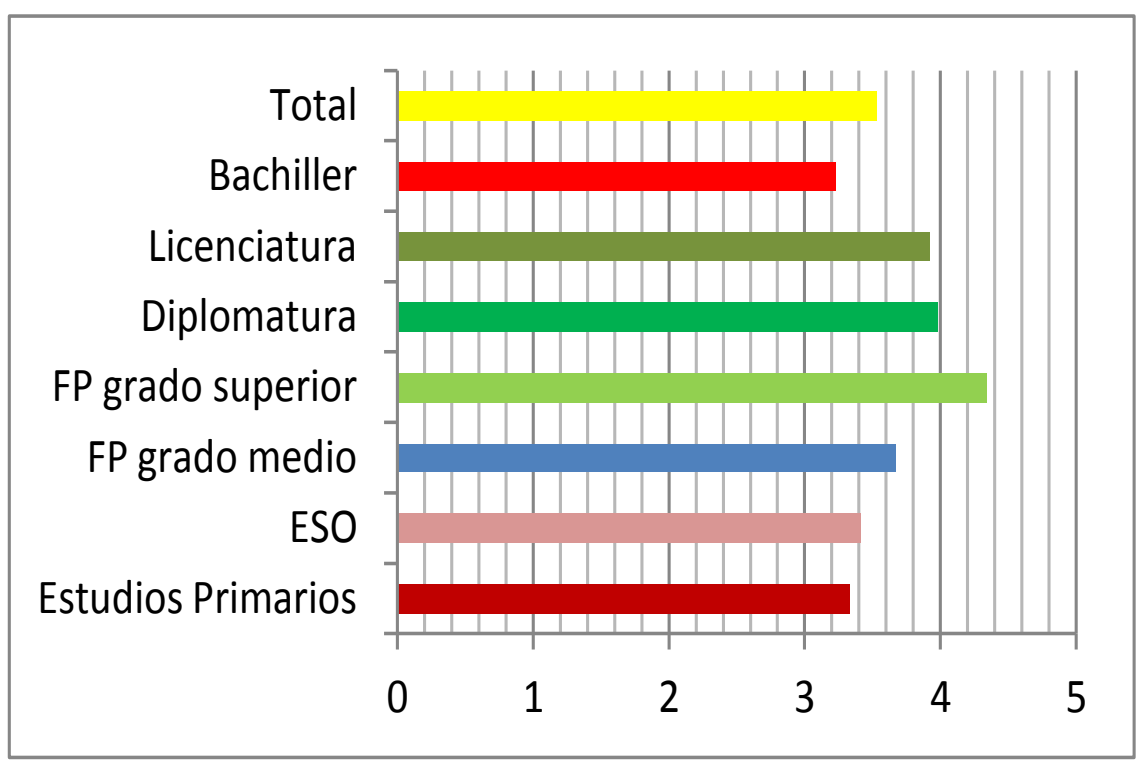

Figura 1. Opinión de padres/madres según los estudios realizados 
-Población de residencia:

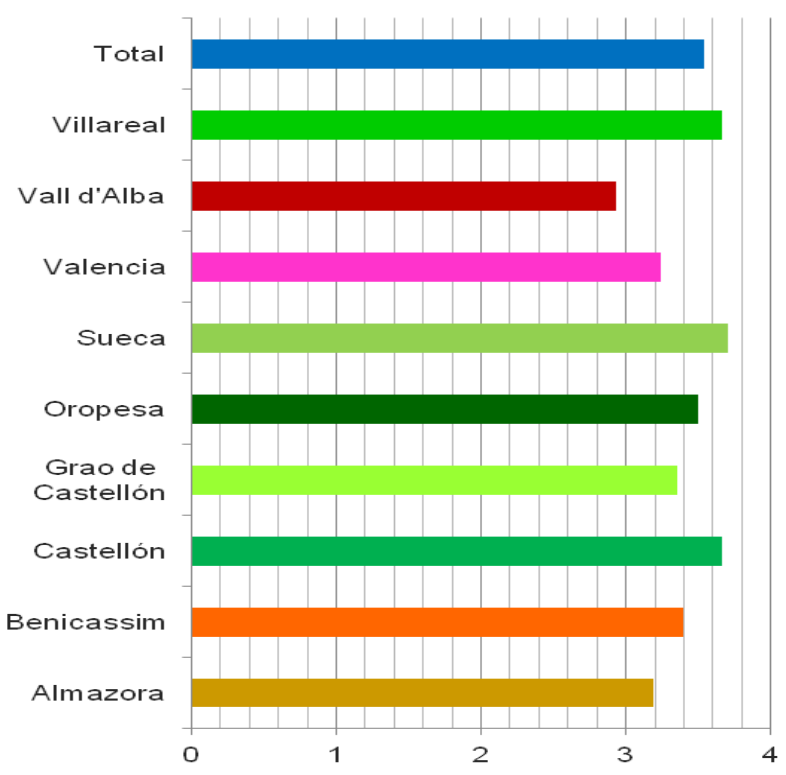

- Almazora

- Benicassim

-Castellón

-Grao de Castellón

- Oropesa

- Sueca

- Valencia

-Vall d'Alba

- Villareal

- Total

Figura 2. Opinión de padres/madres según la localidad donde residen

\section{Centro donde estudian los hijos e hijas}

\begin{tabular}{|l|l|l|}
\hline $\begin{array}{l}\text { CENTRO } \\
\text { DONDE } \\
\text { ESTUDIAN } \\
\text { LOS HIJOS }\end{array}$ & $\begin{array}{l}\text { MEDIA DE ITEMS } \\
\text { CARÁCTER } \\
\text { POSITIVO HACIA } \\
\text { DIFICULTADES DE } \\
\text { APRENDIZAJE }\end{array}$ & $\begin{array}{l}\text { MEDIA DE ITEMS } \\
\text { DE CARÁCTER } \\
\text { NEGATIVO HACIA } \\
\text { DIFICULTADES DE } \\
\text { APRENDIZAJE }\end{array}$ \\
\hline Público & 3,47 & 2,83 \\
\hline Privado & 4,30 & 1,66 \\
\hline Concertado & 3,40 & 2,97 \\
\hline Total & 3,53 & 2,77 \\
\hline
\end{tabular}

Figura 3. Según el centro donde estudian los hijos e hijas de los padres encuestados, opinión sobre NEE 


\section{El género}

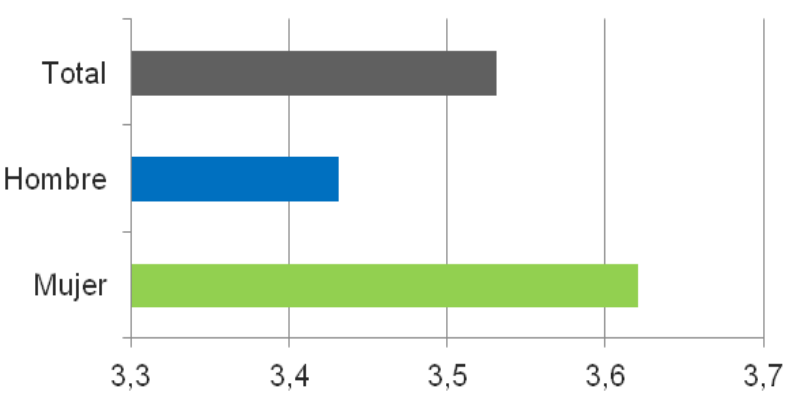

Mujer

-Hombre

-Total

Figura 4. Opinión de NEE según el género de los padres

A continuación expondremos los resultados de los cuestionarios pasados a profesores. Así pues este es el segundo bloque importante del análisis realizado, recordando que el primero ha sido el bloque de "padres y madres". En este apartado también se puntúa entre 1 y 5 , según las siguientes variables:

\section{Según el tipo de centro}

Dónde ejercen su profesión, la información que tienen sobre las NEE:

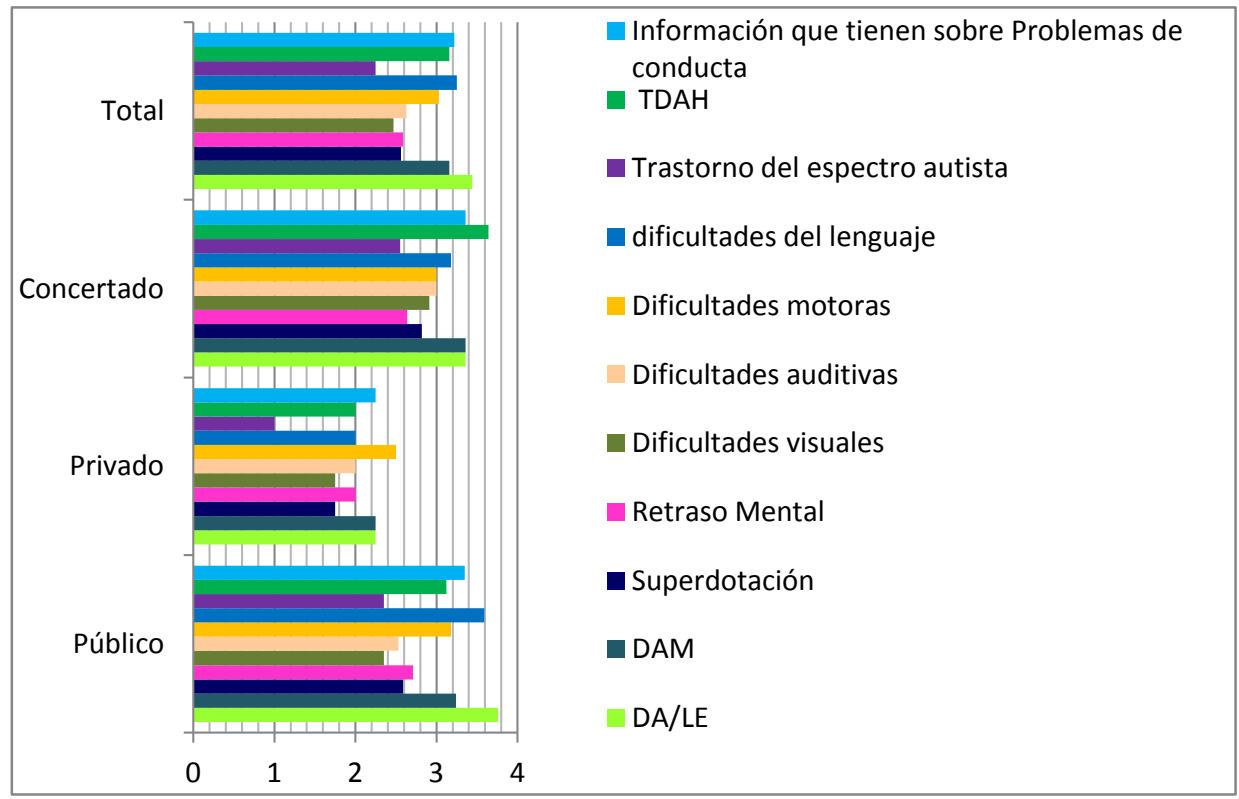

Figura 5. Información que presentan los profesores acerca de las diferentes NEE según el tipo de centro 


\section{-El contacto que han tenido con las distintas NEE:}
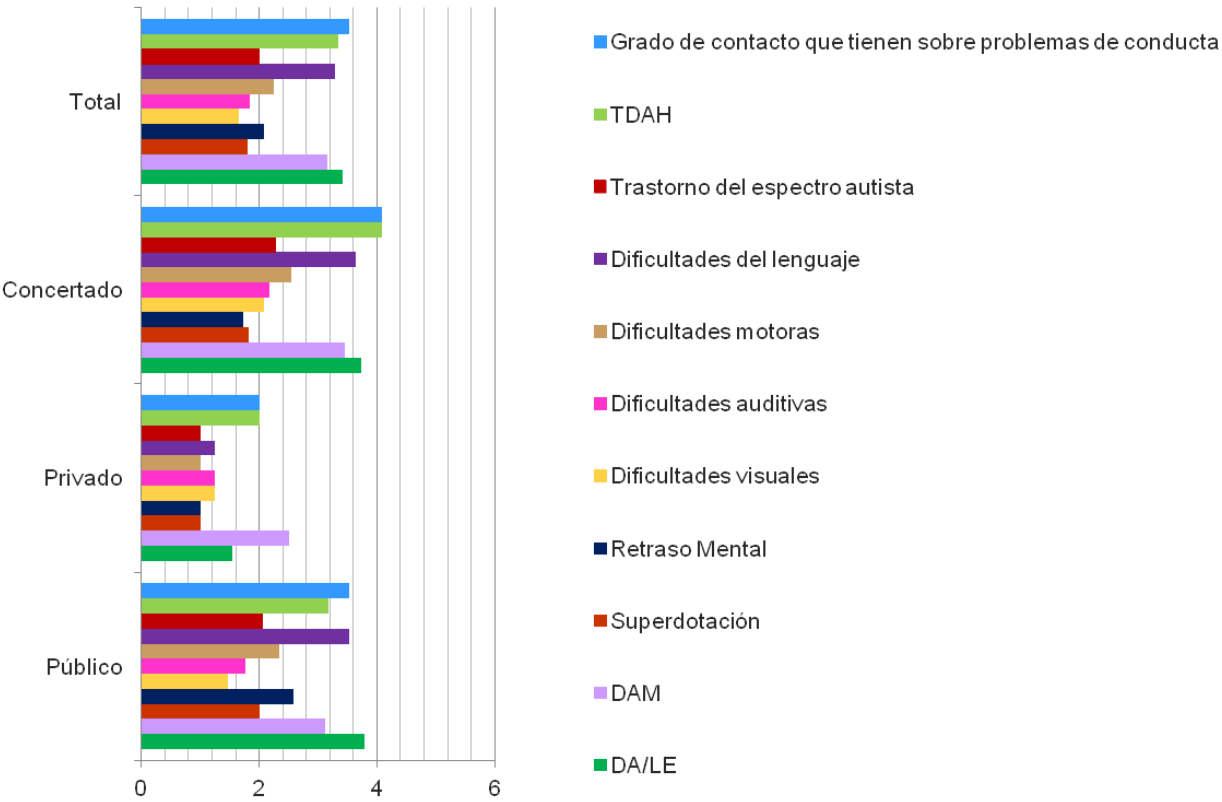

Figura 6. Contacto que los profesores han tenido con las NEE, según la tipología de centro

\section{-El grado de formación que necesitan:}

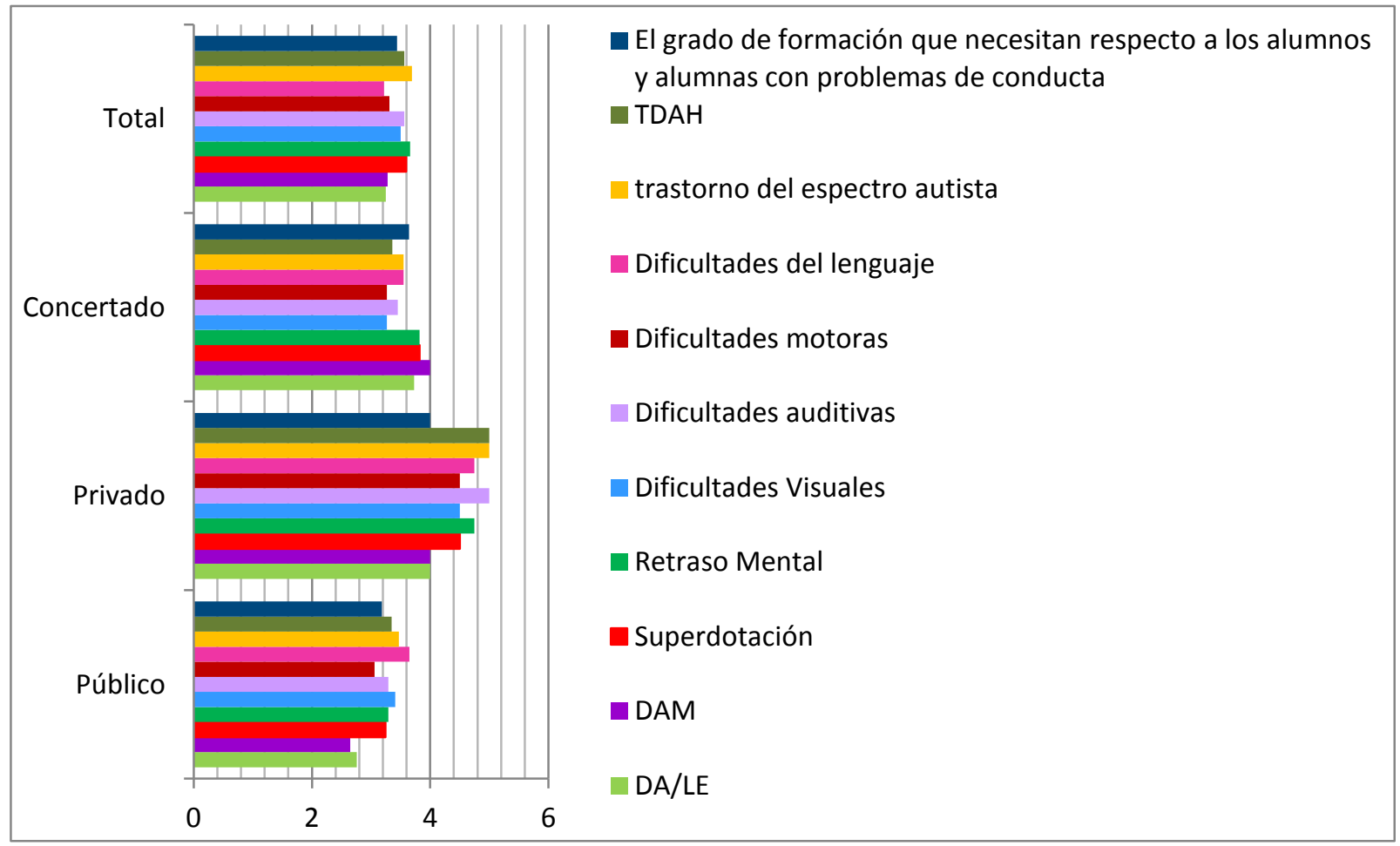

Figura 7. Grado de formación que consideran que necesitan los profesores 


\section{-Predisposición a tener en el aula alumnos y alumnas con dificultades de aprendizaje:}

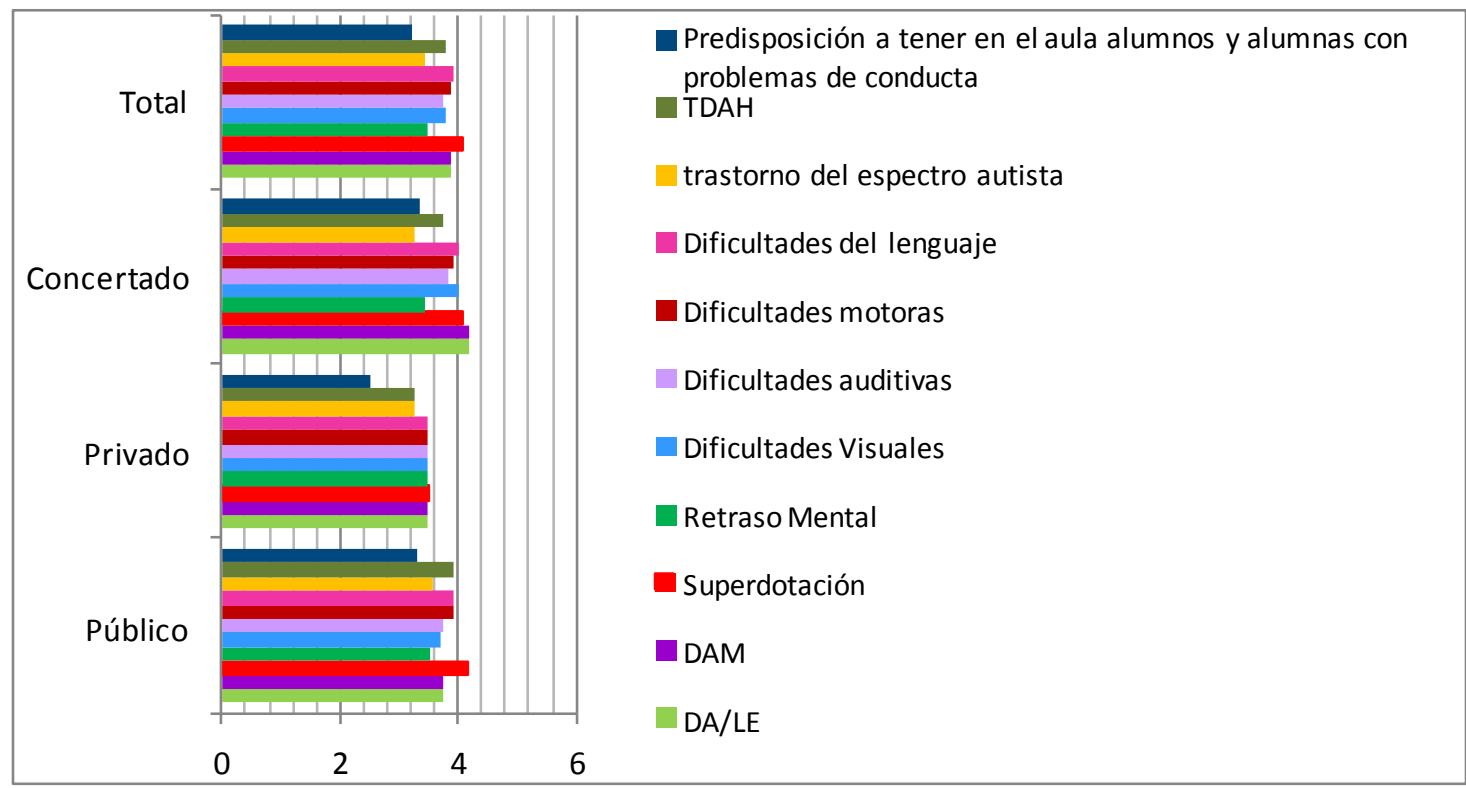

Figura 8. Trato con las diferentes NEE que los profesores han tenido en sus aulas

\section{-El grado de dificultad académica que presentan los niños con las} diferentes NEE:

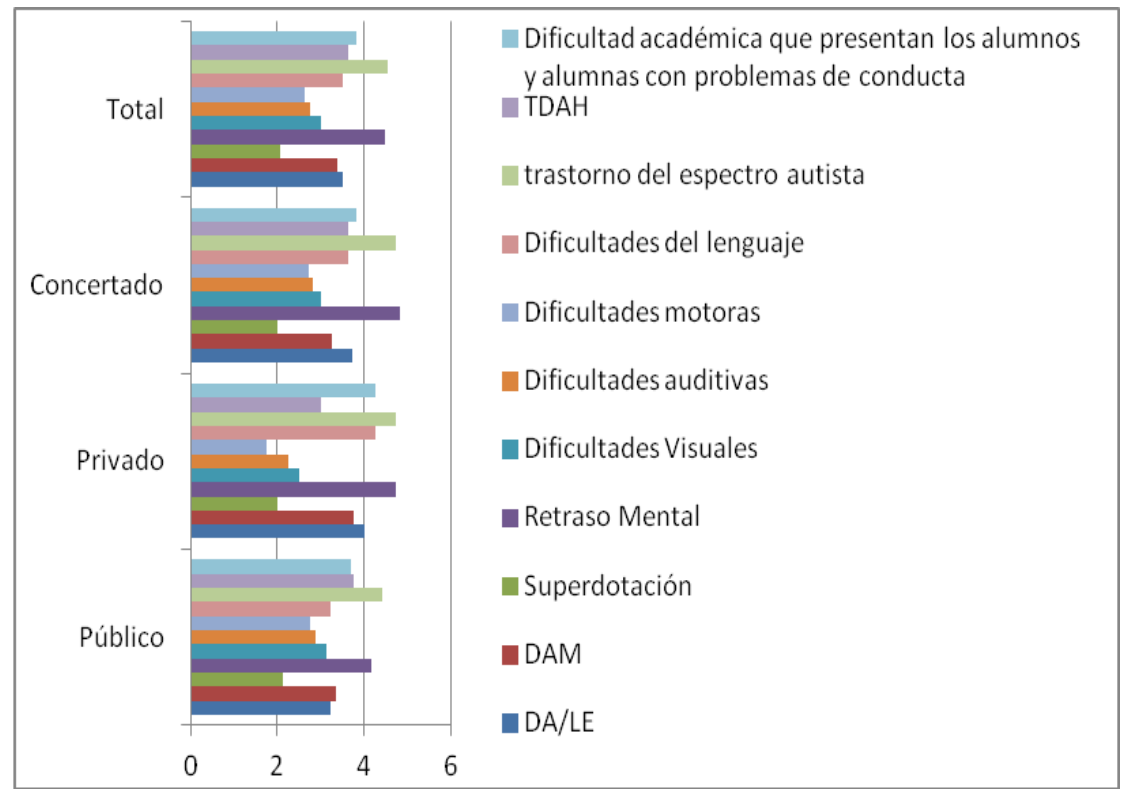

Figura 9. Opinión de profesores según el grado de dificultad académica que presentan los niños con DA 
Según la edad del docente:

-La formación que los maestros y maestras tienen sobre NEE:

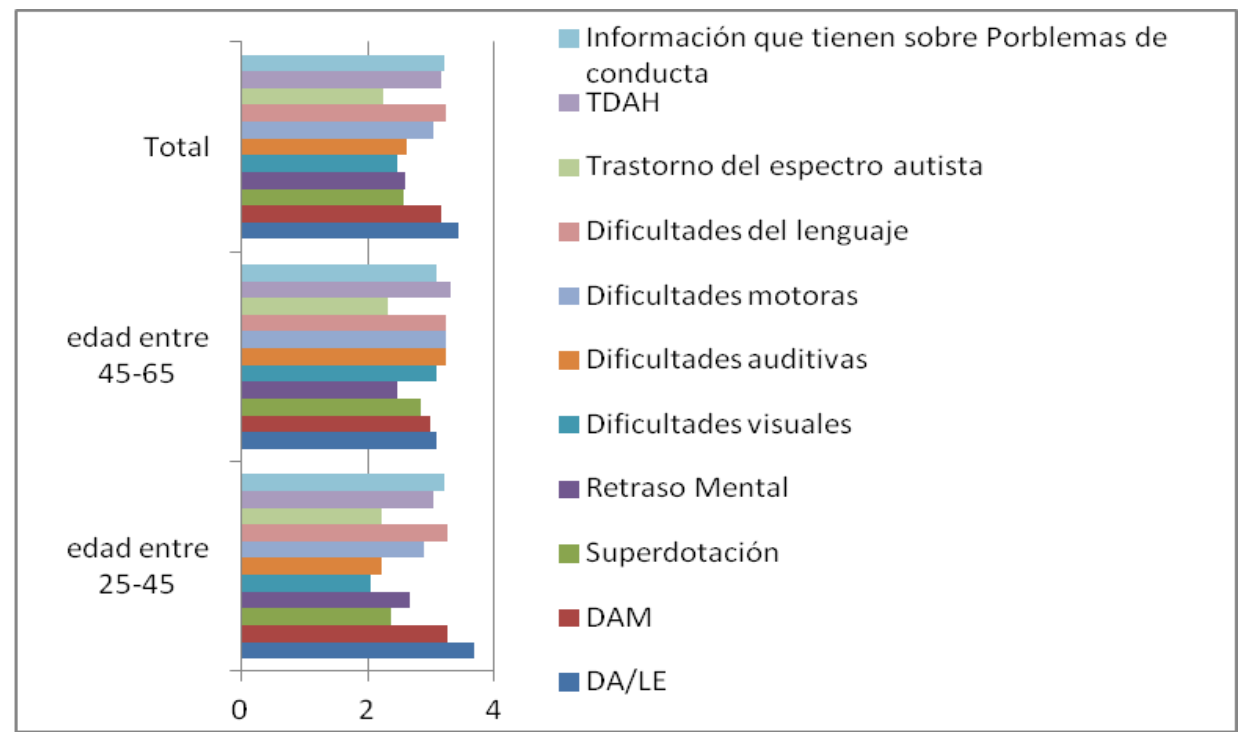

Figura 10. Opinión de profesores según la formación que los maestros y maestras tienen sobre NEE

-Formación sobre NEE que los docentes consideran que necesitan:

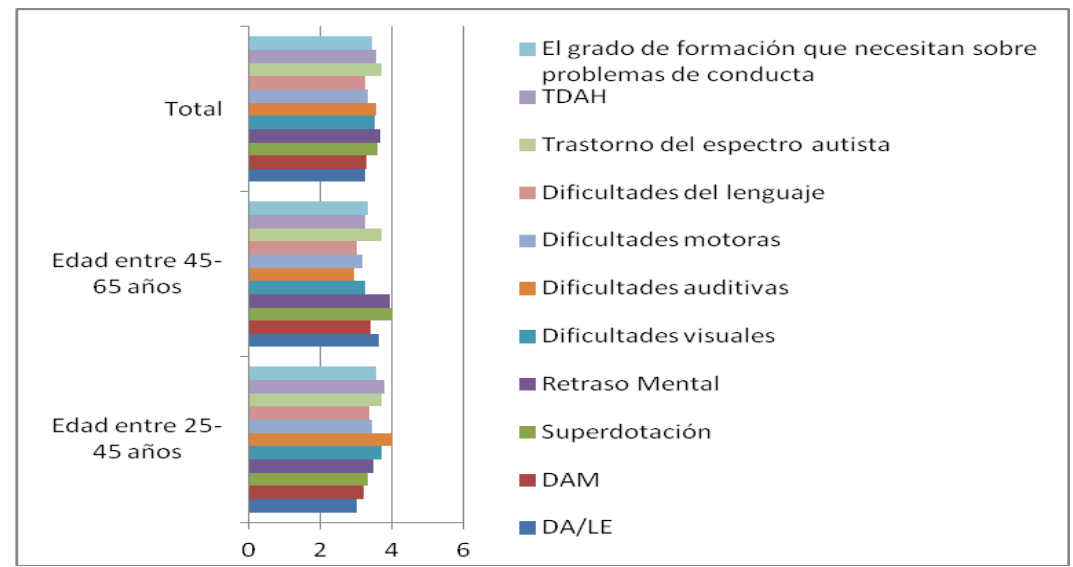

Figura 11. Opinión de profesores según la formación sobre NEE que los docentes consideran que necesitan 


\section{Discusión y conclusiones}

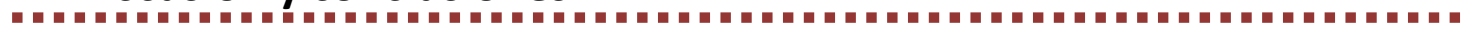

A continuación, presentamos el análisis, discusión e interpretación de los resultados de las encuestas de los padres y madres:

-Según los estudios realizados: Observamos que los padres que tienen actitudes más positivas hacia la inclusión de niños y niñas con NEE son los padres y madres cuyos estudios son FP de grado Superior. En contra de nuestra hipótesis inicial que apuntaba a que cuanto menor era el nivel de estudios, menos positiva sería la actitud.

-Según el centro donde estudian los hijos e hijas: Se ha analizado que hay una actitud más positiva en aquellos padres que llevan sus hijos a colegios privados frente a aquellos que llevan sus hijos a colegios concertados. Este resultado también nos ha llamado la atención ya que pensábamos que los padres que llevaban sus hijos a colegios públicos serían los que más predispuestos a fomentar la inclusión de los niños con NEE en el aula ordinaria, pero ya hemos visto que no es del todo así.

-Según el género: Hay tendencia a que las actitudes más positivas sean por parte de las mujeres, verificando nuestra hipótesis inicial. Esto pensamos que puede deberse a que como las mujeres tienen la posibilidad de ser madres, en la mayoría de los casos, creemos que pueden mostrar una actitud más empática y solidaria referente a los problemas genéticos que pueden afectar. Aunque hay que tener en cuenta que la diferencia entre mujeres y hombres no es abismal, siendo ambas actitudes recogidas bastante positivas.

-Según la población de residencia: Los habitantes de Sueca, Castellón y Villareal son los que presentan más actitudes positivas, frente a Vall d'Alba que es la población que presenta las actitudes menos positivas. Estos resultados irían en contra de nuestra hipótesis, ya que pensábamos que las ciudades o pueblos más grandes tendrían actitudes menos positivas frente a los pueblos más pequeños, donde la inclusión podría ser mucho más positiva debido a la ratio de alumnos/as por maestro y por el supuesto vínculo de afectuosidad y cordialidad que tienen los habitantes de un pueblo medianamente pequeño frente a los habitantes de ciudades o grandes pueblos. De entre las conclusiones e interpretación de los resultados de las encuestas de los profesores y las profesoras, destacamos:

-Según el centro donde ejercen su profesión, la información que tienen sobre las diferentes dificultades de aprendizaje: Podemos observar a modo general que la NEE de la cual los profesores están menos informados 
es el autismo, contrastando así con nuestra hipótesis. El autismo es un trastorno relacionado con la comunicación, más exactamente con la dificultad de establecer comunicación con su entorno, incapacidad de interacción social, que afecta también la imaginación, la planificación y la reciprocidad emocional y evidencia conductas repetitivas o inusuales, por lo tanto la relación profesor/a-alumno/a se hace muy compleja y debe ser estudiada con minuciosidad por parte del primero. Frente a esta necesidad destacan las dificultades en lectura, que son las dificultades de aprendizaje de las que se considera tener más información o se cree que se conocen más a fondo.

Clasificando los centros en privados, concertados y públicos, observamos que los centros privados son los que menos información tienen sobre las NEE, teniendo éstos más conocimiento sobre las dificultades motoras frente al autismo. Los colegios públicos y concertados se mantienen más o menos a la par, aunque en los públicos tienen más información sobre dificultades en la lectura, frente al autismo y las dificultades visuales y en los concertados tienen más información sobre el TDAH frente a al autismo.

Finalmente, podemos destacar que el autismo es la NEE que se considera más desconocida y más lejana a ser tratada de una manera óptima e inclusiva en los centros ordinarios.

-Según el centro analizamos el contacto que han tenido con las diferentes NEE: En este caso observamos que a modo general los colegios concertados son los que han tenido más contacto con alumnos y alumnas con dificultades de aprendizaje, seguido de los públicos, frente a los privados que son los que menos contacto mantienen.

Dentro de los centros públicos la dificultad con la que han tenido más contacto es la relacionada con la lectura frente a las dificultades visuales. $\mathrm{Si}$ observamos los centros privados observamos que la dificultad que más se denota es la relacionada con la matemática frente a la superdotación, el retraso mental, las dificultades motoras y el autismo que se observan con escasa puntuación. Contemplando los colegios concertados vemos que las dificultades con la que han tenido más contacto son el TDAH y problemas de conducta frente al retraso mental. El resultado que nos ha salido en los centros concertados quizá sea el que más se acerca a nuestra hipótesis, ya que consideramos que deberían haber tenido todos los centros más contacto con las diferentes dificultades de aprendizaje, y como mínimo, la misma relación que nos da como resultado en los centros de tipo concertado. Otra hipótesis que barajábamos en esta cuestión era que creíamos que en los colegios públicos existía mayor grado de heterogeneidad del alumnado, pero los datos nos muestran otra realidad. Diferente posibilidad es que sí que haya existido esa diversidad pero no se haya sabido diagnosticar adecuadamente. 
-Según el centro, el grado de formación que necesitan: Dentro de los centros públicos vemos que se considera que necesitan más formación sobre el autismo frente a las dificultades en matemáticas y en lenguaje. En los centros privados, contemplamos que mantienen que necesitan más formación acerca de las dificultades auditivas, el autismo y el TDAH frente al retraso mental. Y por último los colegios concertados basan su necesidad en las dificultades en las matemáticas con un frente a las dificultades visuales y motoras.

En este mismo apartado, los mismos profesores justifican su propia preparación profesional para diagnosticar una NEE. Según observamos en los datos, los centros públicos son los que consideran que no necesitan tanta formación sobre dificultades de aprendizaje, frente a los colegios privados, en los cuales el profesorado considera que necesita mucha más formación sobre el tema. Vemos, a partir de las tablas anteriores, que son éstos los centros que menos contacto han tenido con alumnos y alumnas con dificultades de aprendizaje. Por esta razón, se puede suponer que, como desconocen el tema, no han podido detectar las distintas dificultades de aprendizaje.

-Según el centro, predisposición que se tiende a tener en el aula alumnos y alumnas con dificultades de aprendizaje: Observamos que los centros concertados tienen más predisposición a tener alumnos y alumnas con NEE en sus aulas y casi a la par con los colegios públicos, frente a los centros privados en los cuales parece que hay menos actitud positiva hacia la inclusión (según observamos en los datos). Podemos observar que la dificultad de aprendizaje más aceptada para la predisposición de las NEE en el aula es la superdotación frente a los problemas de conducta.

En los colegios públicos hay más predisposición por la superdotación frente a las dificultades de problemas de conducta. Dentro de los colegios privados vemos que hay predisposición por todas las dificultades de aprendizaje en casi todas por igual, exceptuando en las dificultades de problemas de conducta que proponen un valor inferior. Y dentro de los colegios concertados observamos que hay más predisposición por las dificultades en matemáticas y lenguaje frente a los problemas de conducta.

Así pues y como conclusión, observamos claramente que la dificultad de aprendizaje más rechazada en el aula es la relacionada con los problemas de conducta, coincidiendo así en las tres tipologías de centro.

-Según el centro el grado de dificultad académica que presentan los niños con las diferentes NEE: En los colegios públicos consideran que el autismo es la NEE que presenta más dificultad académica frente a la superdotación. En nuestra hipótesis inicial no encaja esta suposición ya que la superdotación en la mayoría de los casos se muestra con un fracaso escolar 
elevado, ya que es una de las NEE menos diagnosticadas y menos intervenida, como se ha observado anteriormente, con lo cual conlleva una elevada dificultad académica. En el caso de los colegios privados observamos que el autismo y el retraso mental son dos casos en las que el alumno/a presenta una mayor dificultad académica frente a las dificultades motoras. Dentro de los colegios concertados vemos que el retraso mental es que el que más dificultades presenta frente a la superdotación, que es la dificultad más asimilada por el profesorado.

-Según el centro, el grado de dificultad social que tienen los alumnos y alumnas con NEE: Observamos que la dificultad de aprendizaje que causa más dificultad social es el autismo frente a la dificultad en el aprendizaje matemático, que es la que menos dificultad presenta.

En los colegios públicos muestran más grado de dificultad social las NEE de autismo junto con los de problemas de conducta frente a las dificultades en lectura y matemáticas. Dentro de los colegios privados vemos que la necesidad educativa que muestra más dificultad social es el retraso mental frente a la superdotación y las dificultades en matemáticas. En los colegios concertados podemos ver que la NEE que presenta más dificultad social es el autismo nuevamente frente a la dificultad en matemáticas.

Como conclusión en este apartado, podemos observar claramente que la problemática que ocasiona menos dificultad social es la relacionada con las matemáticas, coincidiendo así en las tres tipologías de centros también.

-Según la edad, la formación que los maestros y maestras tienen sobre NEE: El grado de formación es más alto en los profesionales de 46 años o más, que en los de 45 años y menores, apoyando así nuestra hipótesis inicial pero pudiendo ir en contra de suposiciones y pensamientos actuales ya que los mayores de 45 años estudiaron antes y se supone que algunas cosas que han aparecido actualmente se desconocían entonces.

Por otro lado, si hacemos una observación más específica vemos que en el primer grupo (25-45 años) tienen más información sobre las NEE relacionadas con la lectura frente a las dificultades visuales. $\mathrm{Y}$ en el segundo grupo (45-65) vemos que tienen más información sobre dificultades lectoras, auditivas y problemas en conducta. Una razón por la que los docentes que se incluyen en este intervalo de mayor edad se consideran mejor formados podría ser porque suelen tener más experiencia y llevan más años en la profesión, y por ello, han tenido mayor posibilidad de encontrarse más casos y han debido aprender a afrontarlos. Por otra parte, no ratifican tener gran experiencia en el caso del autismo.

-Según la edad el grado de contacto que han tenido con las NEE: Grosso modo vemos que los mayores de 45 años han tenido más contacto con 
alumnos y alumnas con NEE que los menores de 45, ya que es lógico pensar que los años que llevan ejerciendo la docencia les posibilita el haber tratado con un número mayor de alumnos que en el caso de los menores de 45.

Analizamos que el primer grupo (25-45) ha tenido más contacto con las NEE relacionadas con la lectura frente a la superdotación. Y en el segundo grupo (45-65) observamos que con la DA que han tenido más contacto es con el TDAH frente al autismo, que es con la que menos contacto se ha tenido.

-Según la edad, el grado de formación sobre NEE que los docentes consideran que necesitan: De manera general apreciamos que el primer grupo (25-45) considera que necesita más formación en cuanto a NEE nos referimos, frente al segundo grupo (45-65), aunque sería conveniente puntualizar que la diferencia entre ambos es mínima. De este modo, coincide cuando el segundo grupo de docentes más experimentados opinan que han tenido más relación con las NEE y que, seguramente, en algún momento hayan tenido que ser autodidactas para aprender a tratar ciertas dificultades que para su tiempo han sido "desconocidas".

Esta investigación nos ha servido para conocer diferentes opiniones acerca de las actitudes que profesores y madres/padres de provincias cercanas a nuestro contexto académico mantienen frente a las NEE.

Respecto a los datos obtenidos, nos llama la atención aspectos como el reconocimiento por parte de los profesores de centros privados, de la escasa formación académica para afrontar y diagnosticar dificultades de aprendizaje en sus aulas, así como su comprensión y positividad hacia la inclusión de alumnos con NEE en las aulas ordinarias. Denotan tener mucha humildad.

Por otro lado, nos ha llamado la atención la actitud positiva hacia la dificultad en el aprendizaje matemático como un problemática superable en un aula ordinaria por parte del profesorado. Asimismo, nos ha sorprendido que el autismo esté todavía tan lejano de ser tratado adecuadamente, ya que pensamos que es necesario, en la formación de un docente, recibir información suficiente para saber trabajar con esta dificultad, sobre todo por la importancia en el ámbito comunicativo que afecta al propio individuo y a su contexto y porque cada vez más las leyes educativas, por lo general, exigen un mayor grado de inclusión en las aulas. Con esta pequeña muestra de encuestados, hemos podido percibir que la sociedad de hoy en día valora la inclusión de dichos niños y niñas en las aulas ordinarias, porque se piensa que como mejor se aprende es entre iguales, de niño a niño, pero su relación escolar no tiene nada que ver con que dispongan distintas capacidades académicas y/o sociales. Aunque por otro lado, se considera que todavía existen perjuicios preestablecidos que coaccionan el posible establecimiento de ambientes más inclusivos en 
nuestra sociedad actual. Hemos podido valorar también, la creencia de que ahora hay más niños con NEE que hace 30 años, pero esto no sería del todo correcto si observamos los porcentajes, hipotéticos, de población con problemáticas de este tipo en épocas anteriores. Sí se puede admitir que ahora existen muchos más métodos y conocimientos para detectar estas necesidades educativas. Así pues, esta podría ser la razón que apoya la creencia de que ahora hay más niños y niñas con NEE: porque se diagnostican mejor.

Indicar finalmente que aunque los resultados de la presente investigación entendemos que no pueden generalizarse, pensamos que deben contribuir a una reflexión sobre la reconstrucción de las concepciones de los profesores a la luz del actual sistema educativo. Además, pensamos que esta contribución debería ser un elemento revitalizador que potencie la inclusión de los alumnos con NEE.

El concepto de educación especial ha estado sometido a diferentes cambios, como no podía ser de otra manera, que afecta no sólo a los aspectos teóricos sino y, sobre todo a la dimensión práctica, a la forma de abordar la inclusión en el aula ordinaria, así como a la forma de abordar la atención a la diversidad desde las diferentes responsabilidades. Es decir, se piensa que los alumnos, todos los alumnos, deben ser considerados como sujetos que en el proceso de enseñanza-aprendizaje deben ser atendidos, de acuerdo con sus características, de una forma individualizada en los centros y aulas, facilitándoseles el aprendizaje de los contenidos curriculares, así como su desarrollo personal, tanto en el centro como en la sociedad.

\section{Bibliografía}

Álvarez, M., et al. (2005): «Actitudes de los maestros ante las necesidades educativas específicas», Psicothema, vol. 17, n . 4, 601-606.

BAILón, A. (2011): «El papel de los padres en el tratamiento de la dislexia», Logopedia y pedagogía, Granada

CHINER, E. (2011): «Las percepciones y actitudes del profesorado hacia la inclusión del alumnado con necesidades educativas especiales como indicadores del uso de prácticas educativas inclusivas en el aula», Tesis doctoral, Universidad de Alicante.

CORREIA, L. M. (1997): Alunos com Necessidades Educativas Especiais nas Classes Regulares, Porto Editora, Porto. 
DAMM, X. (2009): «Representaciones y actitudes del profesorado frente a la integración de niños/as con necesidades Educativas especiales al aula común», Revista latinoamericana de educación inclusiva, 3 (1), 25-35.

DUEÑAS, M.L. (1991): La integración escolar: aproximación a su teoría y a su práctica, UNED, Madrid.

FALCAO, I. J. (1992): Crianças sobredotadas. Que Sucesso Escolar?, Ediçoes ASA, Rio Tinto.

PARRILLA, A. (1992): El profesor ante la Integración Escolar: investigación y formación, Cincel, Buenos Aires.

RICHARDSON, V. (1996): "The role of attitudes and beliefs in learning to teach». En J. Sikula (Ed.), Handbook of research on teacher education, Macmillan, New York.

ROSALES, C. (1988): «El profesor ante la integración de niños deficientes: actitudes, actuación, preparación», Enseñanza \& Teaching. Revista interuniversitaria de didáctica, 4-5, Universidad de Salamanca, Salamanca.

SÁnCHEZ, A. (2011): "Actitud de los profesores y terapeutas ocupacionales en formación hacia las dificultades de aprendizaje y la discapacidad», Resumen de proyecto. Universidad Central. Santiago de Chile.

STAINBACK, W. y STAINBACK, S. B. (1990): Support networks for inclusive schooling: Interdependent integrated education, Paul H. Brookes, Baltimore

UNESCO (1994): «Declaración de Salamanca y marco de acción para las necesidades educativas especiales», París.

UNICEF (2001): "Hacia el desarrollo de escuelas inclusivas». En HINENI, UNESCO y UNICEF. Ciclo de debates: Inclusión de niños con discapacidad en la escuela regular, Santiago de Chile. 
